\title{
Chiral dinuclear Ln(III) complexes derived from S- and R-2-(6-methoxy-2-naphthyl)propionate.
}

\section{Optical and magnetic properties $\uparrow$}

3

4
5

Berta Casanovas, ${ }^{a}$ Saskia Speed, ${ }^{a}$ Mohamed Salah El Fallah, ${ }^{a}$ Ramon Vicente, ${ }^{* a}$ Mercè Font-Bardía, 西

${ }^{\mathrm{b}}$ Francesco Zinna ${ }^{\mathrm{c}}$ and Lorenzo Di Bari ${ }^{\mathrm{c}}$

a Departament de Química Inorgànica i Orgànica, Secció de Química Inorgànica, Universitat de Barcelona, Martí i Franquès 1-11, 08028 Barcelona, Spain.

b Departament de Mineralogia, Cristal-lografia i Dipòsits Minerals and Unitat de Difracció de R-X,

Centre Científic i Tecnològic de la Universitat de Barcelona (CCiTUB), Universitat de Barcelona, Solé i Sabarís 1-3, 08028 Barcelona, Spain

c Dipartimento di Chimica e Chimica Industriale, Università di Pisa, via Moruzzi 3, I-56124 Pisa, Italy

Ramon Vicente: rvicente@ub.edu 
30 ABSTRACT:

31

32 The reaction of $\mathrm{LnCl} 3 \cdot 6 \mathrm{H} 2 \mathrm{O}$ with (S)-(+)-2-(6-methoxy-2-naphthyl)propionic acid (S-HL), best known

33 as naproxen, and 1,10-phenanthroline (phen) in EtOH allows the isolation of dinuclear chiral

34 compounds S-1-4 of the formula [Ln2(S-L)6(phen)2] 3DMF·H2O [ $\mathrm{Ln}(\mathrm{III})=\mathrm{Eu}(1), \mathrm{Gd}(2), \mathrm{Tb}(3)$ and

35 Dy (4)]. The use of the R-enantiomeric species of the HL ligand led to complexes R-1-4 with the

36 formula [Ln2(R-L)6(phen)2]·3DMF $\cdot H 2 \mathrm{O}$. Compounds R- and S-1, 3 and 4 show strong sensitized

37 metal-centred luminescence in the visible region. Moreover, Dy(III) complexes R- and S-4 display field-

38 induced singlemolecule magnet (SMM) behaviour. For chiral and emissive compounds circularly

39 polarized luminescence (CPL) measurements have also been performed.

40 
43 The (S) enantiomer of 2-(6-methoxi-2-naphthyl)propionic acid, best known as naproxen, is one of the most used non-steroidal anti-inflammatory drugs (NSAID) nowadays. Belonging to the phenylalkanoic acid group of NSAIDs, naproxen presents analgesic and antipyretic properties 1 and is commonly used to treat inflammatory diseases such as rheumatoid arthritis, spondylitis and osteoarthritis.2 Moreover, both the R- and S-enantiomeric species of 2-(6-methoxi-2-naphthyl)propionic acid are able to coordinate to metal ions through the carboxylate group. A few examples of chiral coordination compounds with naproxen and transition metals such as $\mathrm{Cu}(\mathrm{II}), 3-10 \mathrm{Co}(\mathrm{II}), 11 \mathrm{Ni}(\mathrm{II}), 12 \mathrm{Zn}(\mathrm{II}), 10,13,14 \mathrm{Ag} 15$ and $\mathrm{Cd}(\mathrm{II}) 10$ can be found in the literature, showing bioactive, 5 antioxidant16 or optical and ferroelectric properties, 10 the latter derived from the chirality induced by the carboxylate. Only Conclusions three lanthanide coordination complexes containing naproxen and 1,10-phenanthroline (phen) with the formula [Ln2(SL1) 6(phen)2] where Ln = Gd(III), Dy(III) and Er(III) have been previously reported.17 Moreover, to the best of our knowledge, there are no reported lanthanide coordination complexes containing the R-2-(6-methoxi-2-naphthyl)propionate enantiomer.

Due to the wide medical applications of NSAIDs it is necessary to develop simple methods to detect their presence in biofluids and in wash-offs from pharmaceutical equipment in its cleaning.18 For this purpose, it is useful to take advantage of the characteristic emissions of Ln(III) metal ions, mainly from $\mathrm{Eu}(\mathrm{III})$ and $\mathrm{Tb}(\mathrm{III})$, sensitized via ligand-to-metal energy transfer processes best known as the "antenna effect'.19,20 Consequently, organic ligands coordinated to Ln(III) ions must have chromophore groups such as in the case of these anti-inflammatory species.

62 Moreover, luminescent lanthanide complexes have presentday applications in materials and biosciences fields.21-25 The addition of chiral properties through the use of enantiomerically pure ligands in these kinds of complexes allows their potential use as nonlinear or circularly polarized luminescent materials, a field currently in upswing.26-29 The reported examples of chiral lanthanide complexes are mainly of monoand dinuclear structures.29-33

Chiral ligands induce chiroptical properties to the $\mathrm{f}-\mathrm{f}$ transitions of $\mathrm{Ln}(\mathrm{III})$ ions. In emission these properties can be measured by the circularly polarized luminescence (CPL) technique, which can be quantified by means of the dissymmetry factor glum (eqn (1)) where IL and IR correspond to the left and right circularly polarized components of the emission.

$$
g_{\text {lum }}=2 \frac{I_{\mathrm{L}}-I_{\mathrm{R}}}{\mathrm{L}_{\mathrm{L}}+I_{\mathrm{R}}}=\frac{\Delta I}{I}
$$


Based on previously reported CPL measurements, glum factors are about 10-4-10-3 for non-aggregate organic molecules and for transition metal complexes.34 Meanwhile for $\operatorname{Ln}(\mathrm{III})$ complexes this parameter increases up to $10-1-1.29,35-37$

On the other hand, $\operatorname{Ln}(\mathrm{III})$ ions are ideal candidates to form complexes that can behave as singlemolecule magnets (SMMs)38 with potential applications in spintronics, 39 high density data storage, 40 and quantum computing.41 SMMs show slow relaxation of the magnetization with an energy barrier (Ea) proportional to the local anisotropy of the $\mathrm{Ln}(\mathrm{III})$ ions that prevents the spin reversal.42 Since $\mathrm{Ln}(\mathrm{III})$ ions present, in general, strong spin-orbit coupling, their intrinsic magnetic anisotropy is extremely sensitive to the shape and nature of the ligand field around the ion.43 With the aim of obtaining new compounds in which chiral, magnetic and luminescence properties coexist, we present herein the structural, magnetic and optical studies of eight new chiral lanthanide coordination complexes derived from the use of the pure enantiomeric R- or S-species of 2-(6-methoxi2-naphthyl)propionic acid (R- and S-HL respectively) and the auxiliary ligand 1,10-phenanthroline (phen). Therefore, here we present the first examples of $\mathrm{Ln}$ (III) coordination complexes with the R-HL ligand. Neutral chelating phen ligands can block two coordination sites per Ln(III) ion and terminate further aggregation or potential polymerization. 44 In addition, phen ligands can also act as an efficient antenna to sensitise the luminescence of the lanthanide ions. The enantiomeric pairs of complexes R/S1-4 show the same molecular formula [Ln2(R-L)6(phen)2] 3DMF-H2O for $\operatorname{Ln}(\mathrm{III})=\mathrm{Eu}(\mathrm{R}-1)$, Gd (R2), Tb (R-3) and Dy (R-4) and [Ln2(S-L)6(phen)2]·3DMF·H2O for Ln(III) = Eu (S-1), Gd (S-2), Tb (S3) and Dy (S-4). It is worth mentioning that these compounds have been obtained at room temperature and pressure, even though Ln(III) coordination compounds are often synthesised via solvothermal methods.17,45-47

The previously reported Gd(III) and Dy(III) coordination compounds with naproxen present the same dinuclear unit formula [Ln2(S-L)6(phen)2] as S-2 and S-4 but a different crystal structure, without solvent molecules.17 Structural studies based on single crystal XRD over the already published Gd(III) and Dy(III) compounds and on powder X-ray diffraction (PXRD) over the Er(III) compound revealed that the $\mathrm{Ln}(\mathrm{III})$ ions inside the dinuclear units are octa-coordinated and they are bridged through four carboxylate ligands in a syn-syn coordination mode. Meanwhile, in R- and S-1-4 the carboxylate ligands present two different coordination modes, two bridging syn-syn carboxylate groups and two chelating-bridging carboxylate groups, and the $\mathrm{Ln}(\mathrm{III})$ ions are nonacoordinated. Magnetic studies on the previously published complexes showed field-induced SMM behaviour for the Dy(III) complex with Ueff $=12.0 \mathrm{~cm}-1$. 


\section{Experimental section}

\section{Starting materials}

$113 \mathrm{LnCl3} \cdot 6 \mathrm{H} 2 \mathrm{O}$ salts [Ln(III) = Eu, Tb, Dy (Strem Chemicals), Gd (Aldrich)], (S)-(+)-2-(6-methoxy-2-

114 naphthyl)propionic acid (TCI), (R)-(-)-2-(6-methoxy-2-naphthyl)propionic acid (Aldrich), KOH

115 (Aldrich) and 1,10-phenantroline (Aldrich) were used as received without further purification.

\section{Spectral and magnetic measurements}

118 The elemental analyses of the compounds were performed at the Serveis Científics i Tecnològics of the

119 Universitat de Barcelona. Infrared spectra (4000-400 cm-1) were recorded from $\mathrm{KBr}$ pellets on a

120 PerkinElmer 380-B spectrophotometer. Solid state fluorescence spectra were recorded on a Horiba Jobin

121 Yvon SPEX Nanolog fluorescence spectrophotometer at room temperature.

CPL spectra were recorded at the Università di Pisa with a home-built CPL spectrofluoropolarimeter under UV irradiation $(\lambda \max =365 \mathrm{~nm}$ ) on quartz plate depositions. The depositions of the complexes were obtained from $\mathrm{CH} 3 \mathrm{CN}$ dispersions. $\mathrm{CH} 3 \mathrm{CN}$ was chosen as a dispersant because it is a poor solvent for compounds S- and R-1, 3 and 4, in this way the complexes are not dissolved during the casting. In order to rule out the occurrence of contributions from linear dichroism/linear birefringence, different spectra recorded after rotating the sample by $90^{\circ}$ around the optical axis or around an axis perpendicular to the optical axis were recorded and compared; all the spectra were averaged.

ECD spectra were recorded at the Università di Pisa with a Jasco J-710 spectropolarimeter on the same samples of compounds S- and R-1, 3 and 4, used for CPL measurements. Several spectra were acquired rotating the sample as described above for CPL.

Magnetic measurements were performed on solid polycrystalline samples in a Quantum Design MPMSXL SQUID magnetometer at the Magnetic Measurements Unit of the Universitat de Barcelona. Pascal's constants were used to estimate the diamagnetic corrections, which were subtracted from the experimental susceptibilities to give the corrected molar magnetic susceptibilities.

\section{X-ray crystallography}

Single-crystals of complexes R- and S-1, 3 and 4 were mounted in air on a D8VENTURE (Bruker) diffractometer with a CMOS detector. The crystallographic data, conditions retained for the intensity data collection and some features of the structure refinements are listed in Table S1. $\uparrow$ All the structures were refined by the least-squares method. Intensities were collected with a multilayer monochromated Mo-K $\alpha$ radiation. Lorentz polarization and absorption corrections were made in all the samples. The structures were solved by direct methods, using the SHELXS-97 computer program,48 and refined by the fullmatrix least-squares method, using the SHELXL-2014 computer program.49 The non-hydrogen atoms were located in successive difference Fourier syntheses and refined with aniso-tropic thermal 
parameters on F2. For hydrogen atoms, isotropic temperature factors have been assigned 1.2 or 1.5 times the respective parent.

\section{General syntheses}

Solvothermal methods have been extensively used to synthesise polymeric compounds with diverse interesting structures although the mechanism is not completely clear so far. In fact, previously published compounds with the formula [Ln2(R-L)6(phen)2] were obtained through solvothermal synthesis. 17 So, in our experiment, we use a straightforward ambient temperature and pressure procedure. The preparation of compounds R- and S-1-4 was achieved via the reaction of the corresponding enantiomerically pure R/S-2-(6-methoxy-2-naphthyl)propionic acid (R/S-HL,1.5 mmol, $0.345 \mathrm{~g})$ with $1.5 \mathrm{mmol}(0.084 \mathrm{~g})$ of $\mathrm{KOH}$ dissolved in $15 \mathrm{~mL}$ of $\mathrm{MeOH}$. The mixture was stirred for one hour. After this, a solution of 1,10-phenantroline (phen, $0.3 \mathrm{mmol}, 0.054 \mathrm{~g}$ ) in $10 \mathrm{~mL}$ of $\mathrm{MeOH}$ and another containing the corresponding $\mathrm{LnCl} 3 \cdot 6 \mathrm{H} 2 \mathrm{O}$ salt $(0.25 \mathrm{mmol} ; 0.092 \mathrm{~g}$ for $\mathrm{Ln}=\mathrm{Eu}, 0.093 \mathrm{~g}$ for $\mathrm{Ln}$ $=\mathrm{Gd}, 0.093 \mathrm{~g}$ for $\mathrm{Ln}=\mathrm{Tb}$ and $0.094 \mathrm{~g}$ for $\mathrm{Ln}=\mathrm{Dy}$ ) in $5 \mathrm{~mL}$ of DMF were added. The resulting mixture was stirred for another hour at room temperature. R-HL and $\mathrm{Ln}=\mathrm{Eu}$ for R-1, S-HL and $\mathrm{Ln}=\mathrm{Eu}$ for S-1, $\mathrm{R}-\mathrm{HL}$ and $\mathrm{Ln}=\mathrm{Gd}$ for R-2, S-HL and $\mathrm{Ln}=\mathrm{Gd}$ for S-2, R-HL and $\mathrm{Ln}=\mathrm{Tb}$ for R-3, S-HL and $\mathrm{Ln}=\mathrm{Tb}$ for S-3, R-HL and Ln = Dy for R-4, and S-HL and Ln = Dy for S-4. Good crystals suitable for X-ray analysis of complexes R/S-1, 3 and 4 were obtained after one month of slow evaporation. Meanwhile, for complexes R/S-2 polycrystalline solids were obtained. Anal.Calc. (\%) for S-1 C, 61.71; H, 5.18; N, 4.30. Found: C, 61.2; H, 5.2; N, 4.2, calc. (\%) for R-1 C, 61.71; H, 5.18; N, 4.30. Found: C, 61.6; H, 5.3; $\mathrm{N}, 4.4$, calc. (\%) for S-2 C, 61.43; H, 5.15; N, 4.28. Found: C, 61.5; H, 5.2; N, 4.3, calc. (\%) for R-2 C, 61.43; H, 5.15; N, 4.28. Found: C, 61.4; H, 5.2; N, 4.3, calc. (\%) for S-3 C, 61.43; H, 5.15; N, 4.28. Found: C, 61.2; H, 5.1; N, 4.3, calc. (\%) for R-3 C, 61.34; H, 5.15; N, 4.28. Found: C, 61.3; H, 5.2; N, 4.3, calc. (\%) for S-4 C, 61.14; H, 5.13; N, 4.27. Found: C, 60.9; H, 5.1; N, 4.2, calc. (\%) for R-4 C, 61.14; H, 5.13; N, 4.27. Found: C, 61.0; H, 5.0; N, 4.3. Selected IR bands (KBr pellet, cm-1): 3454 (m), 3047-2827 (s), 1673 (m), 1632 (s), 1604 (vs), 1589 (s), 1550 (s), 1486 (m), 1460 (m), 1426 (m) for S-1, 3444 (m), 3047-2819 (m), 1673 (vs), 1633 (s), 1603 (vs), 1587(s), 1556 (s), 1486 (m), 1462 (vs), 1425 (s) for R-1, 3419 (m), 3047-2835 (m), 1673 (s), 1635 (s), 1604 (vs), 1589 (s), 1558 (m), 1486 (m), 1458 (m), 1425 (s) for S-2, 3443 (m), 3047-2835 (m), 1671 (s), 1631 (s), 1603 (vs), 1584 (s), 1553 (m), 1485 (m), $1456(\mathrm{~m}), 1423(\mathrm{~m})$ for R-2, 3446 (m), 3057-2838 (s), 1673 (s), 1633 (s), 1604 (s), 1589 (s), 1553 (s), $1486(\mathrm{~s}), 1450$ (s), 1426 (s) for S-3, 3448 (m), 3057-2835 (s), 1672 (s), 1631 (s), 1604 (vs), 1588 (s), $1552(\mathrm{~s}), 1482(\mathrm{~m}), 1460(\mathrm{~m}), 1425(\mathrm{~s})$ for R-3, $3443(\mathrm{~m}), 3055-2827(\mathrm{~s}), 1671(\mathrm{~s}), 1633(\mathrm{~m}), 1604$ (vs), $1588(\mathrm{~m}), 1554(\mathrm{~m}), 1485(\mathrm{~m}), 1459(\mathrm{~m}), 1425(\mathrm{~s})$ for S-4 and $3443(\mathrm{~m}), 3047-2835(\mathrm{~s}), 1671(\mathrm{~s})$, 1633 (m), 1604 (vs), 1585 (m), 1554 (m), 1485 (s), 1459 (m), 1425 (s) for R-4. 


\section{X-ray crystal structures}

183 Single-crystal X-ray analysis of complexes R- and S-1, 3 and 4 reveals that all the complexes crystallize 184 in the non-centrosymmetric triclinic P1 space group (Table S1 $\dagger$ ). The asymmetric unit of R- and S-1, 3 and 4 is formed by [Ln2(R/S-L)6(phen)2]·3DMF·H2O units. Based on the elemental analysis and the infrared and powder XRD spectra (Fig. S1 $\uparrow$ ), it is proposed that complexes R- and S-2 are isostructural to the rest of the obtained complexes. Besides the optical isomerism, R- and S-1-4 exhibit the same structure with only slight differences in the structural parameters due to the incremental ionic radius and thus only the structure of compound S-3 will be discussed. [Tb2(S-L)6(phen)2] 3DMF·H2O (S-3). A labelled plot of the structure of the dinuclear fragment of compound S-3 is represented in Fig. 1a. Selected bond distances for R- and S-1, 3 and 4 are listed in Table 1. The structure consists of dinuclear molecules in which each $\mathrm{Tb}(\mathrm{III})$ is nona-coordinated.

In each unit the two $\mathrm{Tb}(\mathrm{III})$ atoms are bridged through four deprotonated carboxylate S-L ligands. There are two different kinds of coordination modes for the carboxylate ligands. One bridging carboxylate in the coordination mode $\mu-1 \kappa \mathrm{O}: 2 \kappa \mathrm{O}^{\prime}$ with the bond lengths $\mathrm{Tb} 1-\mathrm{O} 1, \mathrm{~Tb} 1-\mathrm{O} 5, \mathrm{~Tb} 2-\mathrm{O} 2$, and $\mathrm{Tb} 2-\mathrm{O} 6$ being 2.339(5), 2.353(5), 2.356(5) and 2.367(5) $\AA$, respectively. The second kind of bridging coordination mode of the carboxylate is $\mu-1 \kappa\left(\mathrm{O}, \mathrm{O}^{\prime}\right): 2 \kappa\left(\mathrm{O}^{\prime}\right)$, in which $\mathrm{O} 3$ and $\mathrm{O} 7$ act as bridges between the two Tb atoms with distances of 2.499(5) for Tb1-O3, 2.348 (5) $\AA$ for Tb2-O3, 2.335(5) for Tb1-O7 and 2.552(5) $\AA$ for $\mathrm{Tb} 2-\mathrm{O} 7$, meanwhile $\mathrm{O} 4$ and $\mathrm{O} 8$ are bonded only to one $\mathrm{Tb}(\mathrm{III})$ atom with distances

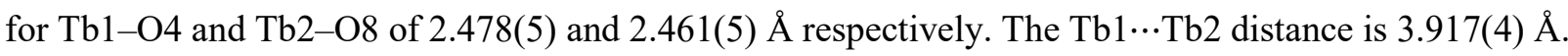
The coordination sphere of each metal is completed by two $\mathrm{N}$ atoms of a 1,10-phenanthroline ligand with bond lengths of Tb1-N1, Tb1-N2, Tb2-N3 and Tb2-N4 of 2.618(6), 2.548(5), 2.582(7) and 2.581(7) A, respectively, and by the two oxygen atoms of a chelating L carboxylate ligand in the $\kappa\left(\mathrm{O}, \mathrm{O}^{\prime}\right)$ coordination mode with bond lengths for Tb1-O9, Tb1-O10, Tb2-O11 and $\mathrm{Tb} 2-\mathrm{O} 12$ of 2.410(5), 2.496(5), 2.484(6) and 2.443(6) $\AA$, respectively. The shortest $\mathrm{Tb} \cdots \mathrm{Tb}$ intermolecular distance in the complex S-3 is 9.840(1) $\AA$ and corresponds to Tb1 $\cdots \mathrm{Tb} 2^{\prime}(': 1+\mathrm{x}, \mathrm{y}, \mathrm{z})$, the rest of the shortest $\mathrm{Ln} \cdots \mathrm{Ln}$ intermolecular distances are collected in Table S2. $\dagger$ LnN2O7 arrangements for R- and S-1, 3 and 4 are intermediate between various coordination polyhedra geometries (Table S3 in the ESI $\dagger$ ). The lowest shape measures for S-3 correspond to Muffin (MFF-9) and spherical capped square antiprism (CSAPR-9) with values of 1.151 and 1.529 for Tb1 and 1.464 and 1.613 for $\mathrm{Tb} 2$, respectively. In Fig. $1 \mathrm{~b}$ the coordination sphere of the $\mathrm{Tb}(\mathrm{III})$ ions in the $\mathrm{S}-3 \mathrm{Compound}$ is shown. 
214 Three DMF molecules and one water molecule co-crystallize with complexes R- and S-1, 3 and 4. The

215 interaction of the water molecule with the dinuclear entities via a hydrogen bond depends on the enantiomer. Therefore, for the enantiomeric species derived from the S-HL, the atom $\mathrm{H} 1 \mathrm{~W}$ of the water molecule is bonded by the oxygen O11 from one of the chelating S-L ligands. On the other hand, for the $\mathrm{R}$-species of complexes 1, 3 and 4 the water molecule interacts with the $\mathrm{O} 9$ oxygen atom from the other terminal R-L ligand. In both enantiomeric isomers the water molecule forms another hydrogen bond with one of the oxygen atoms from a DMF molecule (Fig. S2 $\dagger$ ). The structural parameters for these supramolecular contacts in R- and S-1, 3 and 4 are summarized in Table S4.†

All compounds R- and S-1, 3 and 4 show intermolecular $\pi$-stacking interactions between the aromatic rings of the phen auxiliary ligands containing the N1 and N2 nitrogen atoms with the ones containing the N3 and N4 nitrogen atoms from an adjacent dinuclear entity. These supramolecular interactions result in a 1D supramolecular structure along the [100] direction, represented in Fig. S3. $\uparrow$ The structural parameters of these contacts are summarized in Table S5. $\uparrow$

Lanthanide contraction on compounds R- and S-1, 3 and 4 is confirmed by the diminution of $\mathrm{Ln}(\mathrm{III})-\mathrm{O}$ and $\operatorname{Ln}(\mathrm{III})-\mathrm{N}$ bond lengths and also on $\operatorname{Ln} 1 \cdots \operatorname{Ln} 2$ intramolecular distances.

The analysis of the structural parameters between each enantiomeric pair reveals that the differences between the hydrogen bonds promote shorter Ln1-O distances compared to the Ln2-O ones and more opened angles for Ln1-O3-Ln2 than for Ln1-O7-Ln2 in the S-enantiomers. These observed tendencies are reversed for the R-enantiomeric species.

If we compare the structure of S-2 and S-4 with the previously reported Gd(III) and Dy(III) coordination compounds derived from naproxen, they present the same dinuclear unit formula [Ln2(S-L)6(phen)2] but a different crystal structure and solvent molecules. Structural studies based on single crystal XRD over the already published Gd(III) and Dy(III) compounds and on powder X-ray diffraction (PXRD) over the $\operatorname{Er}(\mathrm{III})$ compound revealed that the $\mathrm{Ln}$ (III) ions inside the dinuclear units are octa-coordinated and they are bridged through four carboxylate ligands in a $\mu-1 \kappa \mathrm{O}: 2 \kappa \mathrm{O}^{\prime}$ coordination mode. Meanwhile, in R- and S-1-4 the carboxylate ligands present two different bridging coordination modes, two bridging $\mu-1 \kappa \mathrm{O}: 2 \kappa \mathrm{O}^{\prime}$ carboxylate groups and two $\mu-1 \kappa\left(\mathrm{O}, \mathrm{O}^{\prime}\right): 2 \kappa\left(\mathrm{O}^{\prime}\right)$ carboxylate groups and the $\mathrm{Ln}(\mathrm{III})$ ions are nonacoordinated. This change of coordination is reflected in the magnetic properties of the complexes.

\section{Magnetic properties}

Due to the identical magnetic behaviour between the R- and S-enantiomers of complexes 1-4, solidstate direct-current $(\mathrm{dc})$ magnetic susceptibility $(\chi \mathrm{M})$ measurements were performed only on one of the corresponding enantiomers. Powder samples of complex S-1 were measured under an applied magnetic 
$\chi$ MT products versus $\mathrm{T}$ in Fig. 2a. Magnetization dependence on the applied field at $2 \mathrm{~K}$ for compounds $\mathrm{S}-1, \mathrm{~S}-2, \mathrm{~S}-3$ and S-4 was also recorded and is shown in Fig. $2 \mathrm{~b}$.

Room-temperature values of $\chi \mathrm{MT}$ are 2.81 (S-1), $16.18(\mathrm{~S}-2), 23.69(\mathrm{~S}-3)$ and $28.62(\mathrm{~S}-4) \mathrm{cm} 3 \mathrm{~mol}-1$ $\mathrm{K}$. Based on the expected values for two isolated $\mathrm{Ln}(\mathrm{III})$ ions, the following $\chi \mathrm{MT}$ values were calculated:50 Eu(III) ground state 7F0, $\chi \mathrm{MTcalcd}=0 \mathrm{~cm} 3 \mathrm{~K} \mathrm{~mol}-1 ; \mathrm{Gd}(\mathrm{III})$ ground state $8 \mathrm{~S} 7 / 2, \mathrm{gJ}=2$, $\chi \mathrm{MTcalcd}=15.75 \mathrm{~cm} 3 \mathrm{~K} \mathrm{~mol}-1$; Tb(III) ground state 7F6, gJ = 3/2, $\chi \mathrm{MTcalcd}=23.64 \mathrm{~cm} 3 \mathrm{~K} \mathrm{~mol}-1$; Dy(III) ground state $6 \mathrm{H} 15 / 2, \mathrm{gJ}=4 / 3, \chi \mathrm{MT}$ calcd $=28.34 \mathrm{~cm} 3 \mathrm{~K}$ mol -1 . The experimental $\chi \mathrm{MT}$ values

255

and the equation of the values of $\chi \mathrm{M}$ as a function of the temperature is:

$$
\begin{aligned}
Z \mathrm{M}= & \left(N \rho^{2} / 3 k T x\right)\left[24+(27 x / 2-3 / 2) \mathrm{e}^{-x}+(135 x / 2-5 / 2) \mathrm{e}^{-3 x}\right. \\
& +(189 x-7 / 2) \mathrm{e}^{-6 x}+(405 x-9 / 2) \mathrm{e}^{-10 x} \\
& \left.+(1485 x / 2-11 / 2) \mathrm{e}^{-14 x}+(2457 x / 2-13 / 2) \mathrm{e}^{-21 x}\right] / \\
& {\left[1+3 \mathrm{e}^{-x}+5 \mathrm{e}^{-9 x}+7 \mathrm{e}^{-6 x}+9 \mathrm{e}^{-10 x}+11 \mathrm{e}^{-15 x}+13 \mathrm{e}^{-211}\right] }
\end{aligned}
$$

266

with

$$
x=\lambda / k T
$$

269

270

The best fitting value parameter obtained by using eqn (2) was $\lambda=341 \mathrm{~cm}-1$. 
271 For the dinuclear Gd(III) compound S-2, the $\chi$ MT product remains almost constant down to $\sim 20 \mathrm{~K}$ and

272 then decreases to $12.87 \mathrm{~cm} 3 \mathrm{~mol}-1 \mathrm{~K}$ at $2.0 \mathrm{~K}$, suggesting a moderately weak antiferromagnetic

273 exchange interaction. For this complex, fitting of the experimental data was performed by means of eqn

274 (3) using the PHI computer program.54 The best-fit parameters obtained were $\mathrm{J}=-0.03 \mathrm{~cm}-1$ and $\mathrm{g}=$

2752.03 with $\mathrm{R}=4.18 \times 10-5$, confirming the existence of an antiferromagnetic coupling between the metal 276 centres. The J value is comparable with those found for similar Gd(III) homodinuclear compounds.44,55

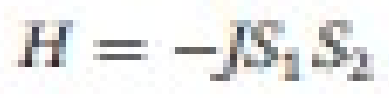

For $\mathrm{Tb}(\mathrm{III})$ (S-3) and $\mathrm{Dy}(\mathrm{III})$ (S-4) compounds the value of the $\chi \mathrm{MT}$ product remains almost constant down to $\sim 50 \mathrm{~K}$ and then decreases to $20.25 \mathrm{~cm} 3 \mathrm{~mol}-1 \mathrm{~K}$ at $7.0 \mathrm{~K}$ for S-3 and to $23.63 \mathrm{~cm} 3 \mathrm{~mol}-1 \mathrm{~K}$ at $6 \mathrm{~K}$ for S-4. From these temperatures the $\chi \mathrm{MT}$ values fall on cooling reaching values of $15.74 \mathrm{~cm} 3$ mol-1 K and $18.62 \mathrm{~cm} 3 \mathrm{~mol}-1 \mathrm{~K}$ for S-3 and S-4, respectively, at $2 \mathrm{~K}$. Magnetization dependence on the applied static magnetic field at $\mathrm{T}=2 \mathrm{~K}$ for complexes S-1, S-2, S-3 and S-4 (Fig. 2b) reveals saturation at high fields only in the case of the Gd(III) complex S-2, with a value of $14.38 \mathrm{~N} \mu \mathrm{B}$ at $5 \mathrm{~T}$.

\section{Ac magnetic susceptibility studies}

For compounds S-3 and S-4 dynamic magnetic studies were performed in order to study if they show

SMM behaviour. The measurements, under an ac magnetic field of $4 \times 10-4 \mathrm{~T}$, reveal that at zero static external magnetic field none of the complexes show in-phase $\left(\chi^{\prime} M\right)$ and/or out-of-phase $\left(\chi^{\prime \prime} M\right)$ ac susceptibility signals under frequencies up to $1488 \mathrm{~Hz}$. This fact may indicate low magnetic anisotropy or that the quantum tunnelling of the magnetization (QTM) process dominates the magnetization relaxation time $(\tau)$, but this relaxation path can be suppressed (or partially suppressed) at low temperatures when a static magnetic field is applied.56

In the case of compound S-3 even under an applied de field of $0.15 \mathrm{~T}$ no frequency or thermal dependency of $\chi^{\prime} \mathrm{M}$ and/or $\chi^{\prime \prime} \mathrm{M}$ is observed.

For complex S-4 the frequency dependence of $\chi^{\prime \prime} \mathrm{M}$ under a zero applied static dc field indicates no maximum in the signal. Under a non-zero field, compound S-4 reveals temperature dependent peaks in the presence of a $0.2 \mathrm{~T}$ dc field, in the temperature range of $1.8-7.0 \mathrm{~K}$ (Fig. S4 $\dagger$ ).

Ac susceptibility frequency dependences of both $\chi^{\prime} \mathrm{M}$ and $\chi^{\prime \prime} \mathrm{M}$ at temperatures between $1.8-3.2 \mathrm{~K}$ were analysed using the Cole-Cole Model (Fig. 3b, Table S6†). 57 The alpha parameter is a bit high suggesting that there is likely to be more than one relaxation process operating at low temperatures.58 Temperature dependence of the relaxation times ( $\tau$ ) (Fig. $3 \mathrm{c}$ ) follows the Arrhenius law $[\tau=\tau 0$ 
$\exp (\mathrm{Ueff} / \mathrm{kBT})$ ], giving an energy barrier (Ueff) of $4.2 \mathrm{~cm}-1$ and a pre-exponential factor $(\tau 0)$ of $4.9 \times$ $10-5 \mathrm{~s}$. As the Ueff value is so low, we suppose a contribution of a direct process following the equation: $\tau-1=\tau 0-1 \exp (\mathrm{Ueff} / \mathrm{kBT})+\mathrm{AT}$. The best fit parameters are: $\mathrm{Ueff}=4.4 \mathrm{~cm}-1, \tau 0=4.6 \times$ $10-5 \mathrm{~s}$, and $\mathrm{A}=45 \mathrm{~s}-1 \mathrm{~K}-1$. The energy barrier obtained is small, but comparable to that of other dinuclear dysprosium complexes reported in the literature.59,60

On the other hand, the relaxation values obtained for S-4 differ significantly from those of the previously reported Dy(III) S-L complex (Ueff $=12 \mathrm{~cm}-1, \tau 0=2.7 \times 10-6 \mathrm{~s}$ ), 17 but this is not surprising because the Dy(III) ions in each complex present different coordination spheres: in S-4 the Dy(III) ions are nonacoordinated and in the previous complex they are octacoordinated and thus S-4 shows a different crystal-field.

\section{Photo-physical studies}

\section{Luminescence studies}

Solid-state luminescence properties of complexes R- and S-1-4 were studied at room temperature. Since all the enantiomeric pairs present the same luminescent response, only one enantiomer will be discussed (Fig. 4).

The emission spectrum of S-Eu(III) (S-1), measured at $\lambda \mathrm{ex}=355 \mathrm{~nm}$ (Fig. 4a), shows the sensitization of the $5 \mathrm{D} 0 \rightarrow 7 \mathrm{FJ}$ transitions at $577 \mathrm{~nm}(\mathrm{~J}=0), 591 \mathrm{~nm}(\mathrm{~J}=1), 614 \mathrm{~nm}(\mathrm{~J}=2), 650 \mathrm{~nm}(\mathrm{~J}=3)$ and $697 \mathrm{~nm}(\mathrm{~J}=4)$. The 5D0 $\rightarrow$ 7F0 magnetically allowed transition shows only one band, suggesting the existence of only one coordination environment for the $\mathrm{Eu}(\mathrm{III})$ ions in S-1.29,61 The dominant 5D0 $\rightarrow$ 7F2 transition shows signs of splitting due to the ligand field around the ions. This splitting is also observed in the 5D0 $\rightarrow 7 \mathrm{~F} 4$ transition. It is not possible to observe ligand emission down to $500 \mathrm{~nm}$ and therefore, the energy transfer from the ligand to the metal seems to be high. The excitation spectrum measured at $\lambda \mathrm{em}=614 \mathrm{~nm}$, Fig. $4 \mathrm{a}$, shows the absorption bands of the ligands and weak bands corresponding to the metal absorption at $395 \mathrm{~nm}(5 \mathrm{~L} 6 \leftarrow 7 \mathrm{~F} 0), 416 \mathrm{~nm}(5 \mathrm{D} 3 \leftarrow 7 \mathrm{~F} 0), 465 \mathrm{~nm}(5 \mathrm{D} 2 \leftarrow$ $7 \mathrm{~F} 0)$ and at $536 \mathrm{~nm}(5 \mathrm{D} 1 \leftarrow 7 \mathrm{~F} 0)$. The emission spectrum of this compound measured in the metal absorption energy range $(\lambda e x=395 \mathrm{~nm}$, Fig. $4 \mathrm{~b})$ is a lot less intense and also shows ligand centred bands because direct metal absorption is less efficient compared to a ligandto-metal energy transfer process.

The emission spectrum of the Gd(III) complex S-2 (Fig. 4c, $\lambda$ ex $=300 \mathrm{~nm}$ ) reveals only a broad band arising from the organic ligands, centred at $375 \mathrm{~nm}$. The corresponding excitation spectrum (Fig. 4c, $\lambda \mathrm{em}=375 \mathrm{~nm}$ ) exhibits two absorption bands, one at $284 \mathrm{~nm}$ and the other at $337 \mathrm{~nm}$. The spectrum recorded at $77 \mathrm{~K}$ (Fig. S5, $\uparrow$ red spectrum) presents two bands assigned to the emission from the singlet (365 nm, $27397 \mathrm{~cm}-1)$ and triplet (417 nm, $23981 \mathrm{~cm}-1)$ excited states. To investigate which of the 
ligands enhances the luminescence of the lanthanides we also recorded the emission spectra of both ligands. The emission spectrum of S-naproxen (Fig. S6†) shows a broad band from 330 to $422 \mathrm{~nm}$ with a maximum at $353 \mathrm{~nm}$, on the other hand, the emission spectrum of phen (Fig. S7†) shows a five maxima emission from 337 to $500 \mathrm{~nm}$ with the highest maximum at $361 \mathrm{~nm}$. With this it seems that the energy transfer process should be enhanced by the ligand phen. The emission spectrum of the $\mathrm{Tb}(\mathrm{III})$ complex S-3 (Fig. 4d, $\lambda$ ex $=350 \mathrm{~nm}$ ) displays metal-centred luminescence due to the 5D4 $\rightarrow$ 7FJ transitions centred at $480 \mathrm{~nm}(\mathrm{~J}=6), 538 \mathrm{~nm}(\mathrm{~J}=5), 575 \mathrm{~nm}(\mathrm{~J}=4)$ and at $609 \mathrm{~nm}(\mathrm{~J}=3)$. Only absorption bands from the ligands are observed in the excitation spectrum.

The emission spectrum of the Dy(III) complex S-4 measured at $\lambda \mathrm{ex}=350 \mathrm{~nm}$ (Fig. 4e) shows the characteristic Dy(III) emission profile with two main transitions 7F9/2 $\rightarrow 6 \mathrm{H} 15 / 2$ and 7F9/2 $\rightarrow$ 9H13/2 at 479 and $573 \mathrm{~nm}$, respectively. The antenna effect in this complex seems to be less efficient compared to that of compounds S-1 and S-3 since a residual ligand emission band appears below $470 \mathrm{~nm}$. The corresponding excitation spectrum ( $\lambda \mathrm{em}=478 \mathrm{~nm}$, Fig. $4 \mathrm{e}$ ) displays a broad band between $400-450 \mathrm{~nm}$ assigned to $\pi-\pi^{*}$ absorption transitions.

\section{Circular dichroism}

353 Solid state electronic circular dichroism (ECD) spectra of complexes S- and R-1, 3 and 4 are shown in

354 Fig. 5 and display Cotton effects in the $\sim 240-360 \mathrm{~nm}$ range due to the chirality of the complexes.31

Ligand-centred electronic transitions display only very weak ECD bands, revealing low dissymmetry in the ligand arrangement around the metal centres. Thanks to the low rotatory strength of transitions associated with phenanothroline and naproxen ligands, some contributions of the usually weak $\mathrm{f}-\mathrm{f}$ transitions are observed in all the cases giving rise to the emergent spectral manifolds. This situation is similar to the one previously observed in the case of analogous 2-phenylpropionic acid-based complexes. 29

\section{CPL measurements}

In view of chiral and luminescence properties of this family of complexes, circularly polarized luminescence (CPL) measurements were performed on visible-energy emitting compounds S- and R-1, 3 and 4. Only S- and R-1 compounds display CPL signals with low intensity emission bands (Fig. 6), centred at $593 \mathrm{~nm}$ and $617 \mathrm{~nm}$ assigned to 5D0 $\rightarrow$ 7F1 and 5D0 $\rightarrow$ 7F2 transitions, respectively. As a consequence of the low intensity of these bands, it has only been possible to quantify the dissym-metry factor for the $5 \mathrm{D} 0 \rightarrow 7 \mathrm{~F} 2$ transition with glum values equal to $\pm 1 \times 10-3$, positive for the R-enantiomer. 
370 but lower than other polynuclear Eu compounds.33,62,63 In order to study the stabilization of these

371 complexes in solution, $\mathrm{CPL}$ measurements have been performed on $\mathrm{CH} 2 \mathrm{Cl} 2$ dissolution but no emission

372 signals were observed. Therefore, based on these results, it seems that the organic ligands dissociate

373 from the complex in solution. 


\section{Conclusions}

376 The use of the R- and S-enantiomerically pure species of 2-(6-methoxi-2-naphthyl)propionic acid and

377 the 1,10-phenantroline auxiliary ligand has led to homodinuclear chiral compounds R- or S-1-4 with the

378 formula [Ln2(R/S-L)6(phen)2]·3DMF $\cdot H 2 \mathrm{O}[\mathrm{Ln}(\mathrm{III})=\mathrm{Eu}(1), \mathrm{Gd}(2), \mathrm{Tb}(3)$ and Dy (4)]. In the

379 syntheses of the new compounds, we have used a straightforward room temperature and pressure

380 procedure.

381 From the magnetic studies recorded on these complexes, the fitting by the Heisenberg-Dirac-Van Vleck

382 (HDVV) spin Hamiltonian of the $\chi \mathrm{MT}$ product vs. T of Gd(III) compounds reveals a weak

383 antiferromagnetic interaction between the two $\mathrm{Ln}$ (III) within the dinuclear unit. The Dy(III) compounds

384 R- and S-4 display field-induced slow relaxation of the magnetization with discrete effective energy

385 barrier values.

386 Moreover, the solid state luminescence study has revealed sensitization of the $\mathrm{f}-\mathrm{f} \mathrm{Ln}(\mathrm{III})$ transitions in

387 the visible region for compounds R- and S-1 [Eu(III)], R- and S-3 [Tb(III)], and R- and S-4 [Dy(III)].

388 Taking advantage of the chiral and emission properties, CPL measurements have been performed on the

389 visible emitting compounds. For the Eu(III) complexes R- and S-1 the analysis of the data has yielded a

390 glum $= \pm 1 \times 10-3$.

391 Thus, compounds R- and S-1, 3 and 4 display luminescence and chiral properties and can be considered

392 as multifunctional complexes. Furthermore, field-induced SMM properties have been observed for R-

393 and S-4. To the best of our knowledge, compounds R- and S-4 are the first examples of homodinuclear

394 Dy(III) complexes in which chiral, emissive and SMM properties are described. 
397 R. V., B. C., M. S. E. F. and S. S. acknowledge the financial support from Spanish government Grant 398 CTQ2015-63614-P. S. S. also acknowledges the financial support from "Obra Social de la Fundació 399 Bancària La Caixa".

400 
S. Etcheverry, D. Barrio, A. Cortizo and P. A. Williams, J. Inorg. Biochem., 2002, 88, 94-100.

D. Mahendiran, P. Gurumoorthy, K. Gunasekaran, R. Senthil Kumar and A. K. Rahiman, New J. Chem., $2015,39,7895-7911$.

A. L. Abuhijleh and J. Khalaf, Eur. J. Med. Chem., 2010, 45, 3811-3817.

F. Dimiza, F. Perdih, V. Tangoulis, I. Turel, D. P. Kessissoglou and G. Psomas, J. Inorg. Biochem., $2011,105,476-489$.

S. Caglar, E. Adiguzel, B. Sariboga, E. Temel and O. Buyukgungor, J. Coord. Chem., 2014, 67, 670683.

C. Dendrinou-Samara, D. P. Kessissoglou, G. E. Manoussakis, D. Mentzafos and A. Terzis, J. Chem. Soc., Dalton Trans., 1990, 959-965.

C. Dendrinou-Samara, P. D. Jannakoudakis, D. P. Kessissoglou, G. E. Manoussakis, D. Mentzafos and A. Terzis, J. Chem. Soc., Dalton Trans., 1992, 3259.

M. Koman, M. Melnık and T. Glowiak, Inorg. Chem. Commun., 2000, 3, 489-492.

Y. T. Wang, G. M. Tang, W. Z. Wan, Y. Wu, T. C. Tian, J. H. Wang, C. He, X. F. Long, J. J. Wang and

F. Dimiza, A. N. Papadopoulos, V. Tangoulis, V. Psycharis, C. P. Raptopoulou, D. P. Kessissoglou and S. W. Ng, CrystEngComm, 2012, 14, 3802-3812. 4492 .

H. Abu Ali, H. Fares, M. Darawsheh, E. Rappocciolo, M. Akkawi and S. Jaber, Eur. J. Med. Chem., $2015,89,67-76$.

M. Paul, K. Sarkar, J. Deb and P. Dastidar, Chem. - Eur. J., 2017, 23, 5736-5747. Dalton Trans., 2014, 43, 6848. 
F. Dimiza, A. N. Papadopoulos, V. Tangoulis, V. Psycharis, C. P. Raptopoulou, D. P. Kessissoglou and G. Psomas, Dalton Trans., 2010, 39, 4517.

Y.-L. Li, Q.-Y. Liu, C.-M. Liu, Y.-L. Wang and L. Chen, Aust. J. Chem., 2015, 68, 488.

A. V. Egorova, A. V. Anelchyk, I. I. Leonenko, Y. V. Skripinets and V. P. Antonovich, J. Anal. Chem., $2015,70,440-449$.

S. V. Eliseeva and J.-C. G. Bünzli, Chem. Soc. Rev., 2010, 39, 189-227.

A. D’Aléo, F. Pointillart, L. Ouahab, C. Andraud and O. Maury, Coord. Chem. Rev., 2012, 256, 1604 1620.

J. Vuojola and T. Soukka, Methods Appl. Fluoresc., 2014, 2, 012001.

M. D. McGehee, T. Bergstedt, C. Zhang, A. P. Saab, M. B. O'Regan, G. C. Bazan, V. I. Srdanov and A. J. Heeger, Adv. Mater., 1999, 11, 1349-1354.

R. C. Evans, P. Douglas and C. J. Winscom, Coord. Chem. Rev., 2006, 250, 2093-2126.

U. Giovanella, M. Pasini, C. Freund, C. Botta, W. Porzio and S. Destri, J. Phys. Chem. C, 2009, 113, $2290-2295$.

E. Peeters, M. P. T. Christiaans, R. A. J. Janssen, H. F. M. Schoo, H. P. J. M. Dekkers and E. W. Meijer, J. Am. Chem. Soc., 1997, 119, 9909-9910.

S. Dang, J.-H. Zhang, Z.-M. Sun and H. Zhang, Chem. Commun., 2012, 48, 11139.

G. Koeckelberghs, S. Sioncke, T. Verbiest, A. Persoons and C. Samyn, Chem. Mater., 2003, 15, 28702872. Commun., 2015, 51, 592-595. 
C. Lincheneau, C. Destribats, D. E. Barry, J. A. Kitchen, R. D. Peacock and T. Gunnlaugsson, Dalton Trans., 2011, 40, 12056.

J. P. Leonard, P. Jensen, T. McCabe, J. E. O’Brien, R. D. Peacock, P. E. Kruger and T. Gunnlaugsson, J. Am. Chem. Soc., 2007, 129, 10986-10987.

E. M. Sánchez-Carnerero, A. R. Agarrabeitia, F. Moreno, B. L. Maroto, G. Muller, M. J. Ortiz and S. de la Moya, Chem. - Eur. J., 2015, 21, 13488-13500.

F. Zinna, C. Resta, S. Abbate, E. Castiglioni, G. Longhi, P. Mineo and L. Di Bari, Chem. Commun., 2015, 51, 11903-11906.

F. Zinna and L. Di Bari, Chirality, 2015, 27, 1-13.37 J. L. Lunkley, D. Shirotani, K. Yamanari, S.

Lanthanides and Actinides in Molecular Magnetism, ed. R. A. Layfield and M. Murugesu, Wiley-VCH Verlag GmbH \& Co. KGaA, 2015.

L. Bogani and W. Wernsdorfer, Nat. Mater., 2008, 7, 179-186.

D. Aguilà, L. A. Barrios, V. Velasco, O. Roubeau, A. Repollés, P. J. Alonso, J. Sesé, S. J. Teat, F. Luis and G. Aromí, J. Am. Chem. Soc., 2014, 136, 14215-14222.

N. C. Anastasiadis, D. A. Kalofolias, A. Philippidis, S. Tzani, C. Raptopoulou, V. Psycharis, C. J. Milios, A. Escuer and S. P. Perlepes, Dalton Trans., 2015, 44, 10200-10209. Commun., 2017, 85, 52-55. 
The Rare Earth Elements: Fundamentals and Applications, ed. D. A. Atwood, John Wiley \& Sons Ltd, 2005.

481

L. E. Ballentine and D. Griffiths, Am. J. Phys., 1991, 59, 1153-1154.

482

M. Andruh, E. Bakalbassis, O. Kahn, J. C. Trombe and P. Porcher, Inorg. Chem., 1993, 32, 1616-1622.

483

O. Kahn, Molecular Magnetism, VHC Publishers, Inc., USA, 1993, vol. 6.

484

N. F. Chilton, R. P. Anderson, L. D. Turner, A. Soncini and K. S. Murray, J. Comput. Chem., 2013, 34, $1164-1175$.

A. Rohde and W. Urland, Inorg. Chim. Acta, 2006, 359, 2448-2454.

487

W.-H. Zhu, X. Xiong, C. Gao, S. Li, Y. Zhang, J. Wang, C. Zhang, A. K. Powell and S. Gao, Dalton Trans., 2017, 46, 14114-14121.

489

K. S. Cole and R. H. Cole, J. Chem. Phys., 1941, 9, 341-351. 58 Y. Song, A. F. Luo, B. Y. Zhu and A. X. Tian, Aust. J. Chem., 2013, 2, 98-104.

D. N. Woodruff, R. E. P. Winpenny and R. A. Layfield, Chem. Rev., 2013, 113, 5110-5148.

492

S. Y. Wang, W. M. Wang, H. X. Zhang, H. Y. Shen, L. Jiang, J. Z. Cui and H. L. Gao, Dalton Trans., $2016,45,3362-3371$.

E. Kreidt, L. Arrico, F. Zinna, L. Di Bari and M. Seitz, Chem. - Eur. J., 2018, 24, 13556-13564.

N. Koiso, Y. Kitagawa, T. Nakanishi, K. Fushimi and Y. Hasegawa, Inorg. Chem., 2017, 56, 57415747.

497

G. Bozoklu, C. Gateau, D. Imbert, J. Pécaut, K. Robeyns, Y. Filinchuk, F. Memon, G. Muller and M. 


\section{Legends to figures}

503

504 Fig. 1 (a) Partially labelled plot of the dinuclear compound S-3. (b) Coordination polyhedra of Tb(III)

505 ions in compound S-3.

506

507

Fig. 2 (a) $\chi$ MT vs. T plot of complexes S-1-4. Solid lines represent the theoretical fit in compounds S-1

508 and S-2. (b) M vs. H plot of compounds S-1-4.

509

510

Fig. 3 Representation of the frequency dependence of the out-of-phase component of the ac

511 susceptibility under a $0.2 \mathrm{~T}$ dc field (a), Argand plots (b) and magnetization relaxation time, $\ln (\tau)$ vs.

$512 \mathrm{~T}-1$ (c) for S-4. Red line represents the fitting using Orbach and direct relaxation processes.

513

514 Fig. 4 Solid-state excitation (dashed line) and emission (solid line) spectra recorded at r. t. for complexes S-1 (a) and (b), S-2 (c), S-3 (d), and S-4 (e).

516

517

Fig. 5 Solid-state ECD spectra for complexes S- and R-1 (a), 3 (b) and 4 (c).

518

519

Fig. 6 Solid-state CPL spectra for complexes S- and R-1.

520

521 


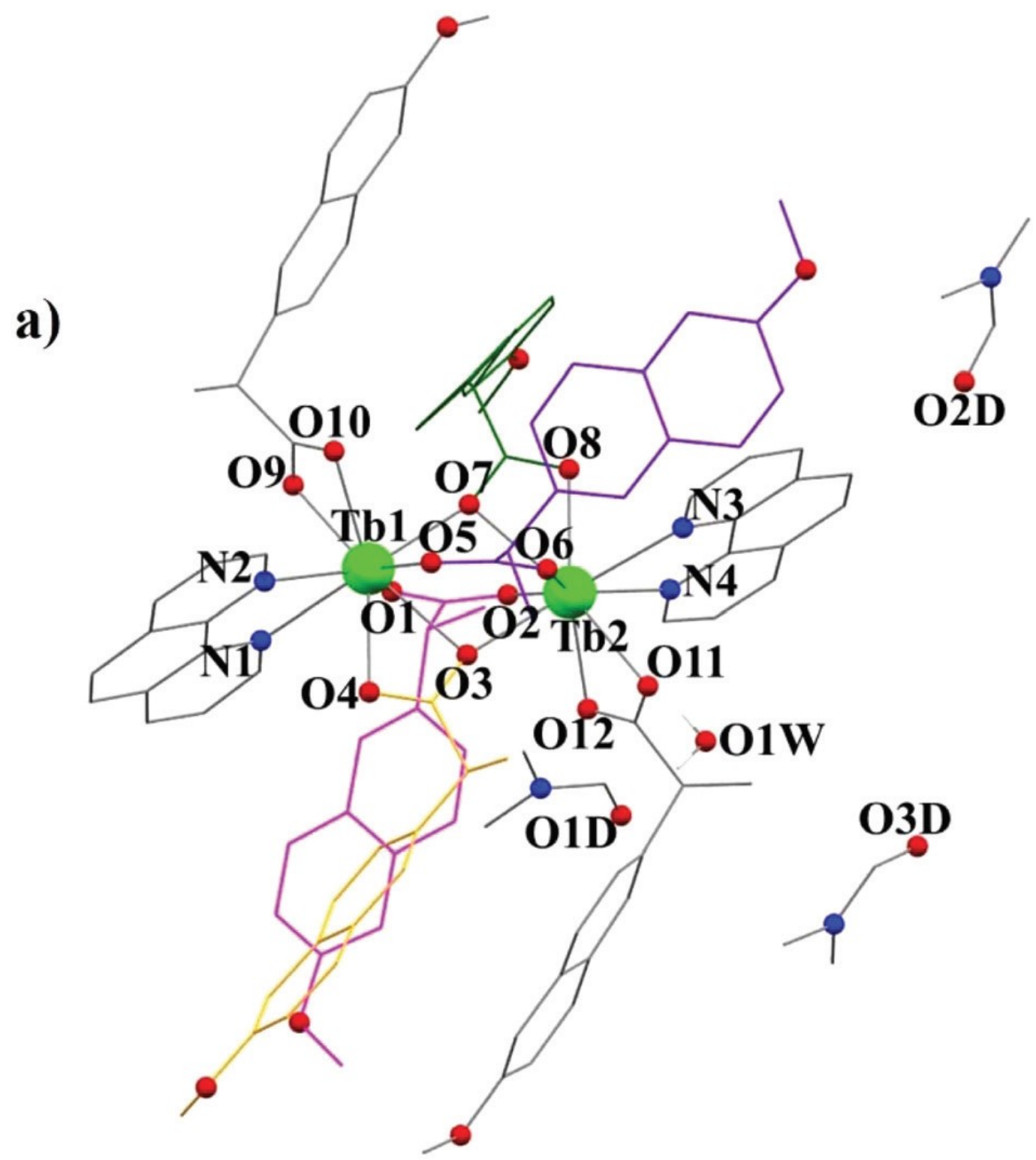

b)

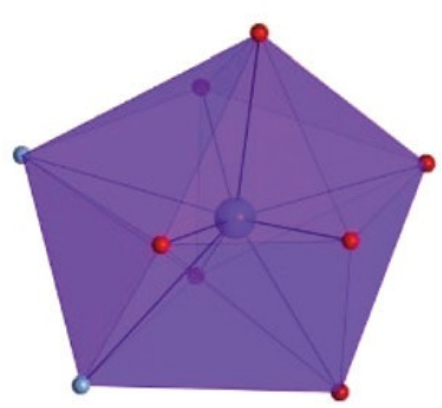

Tb1

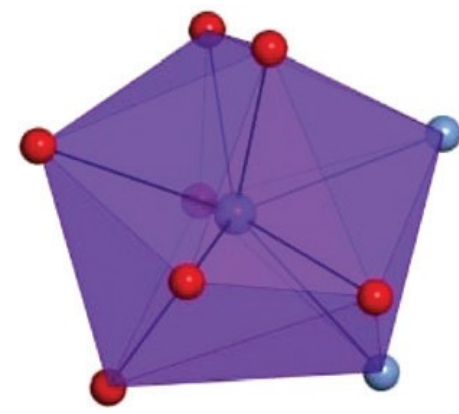

Tb2 

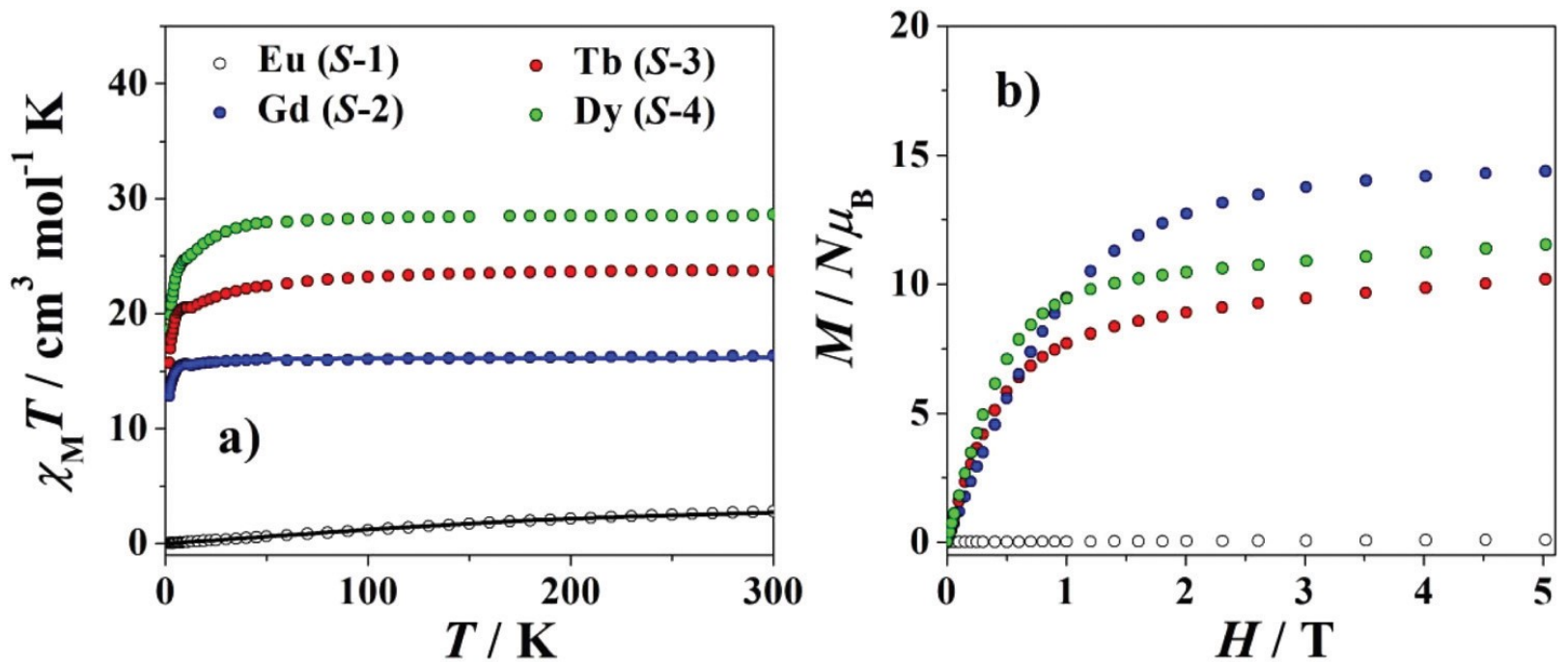

530 

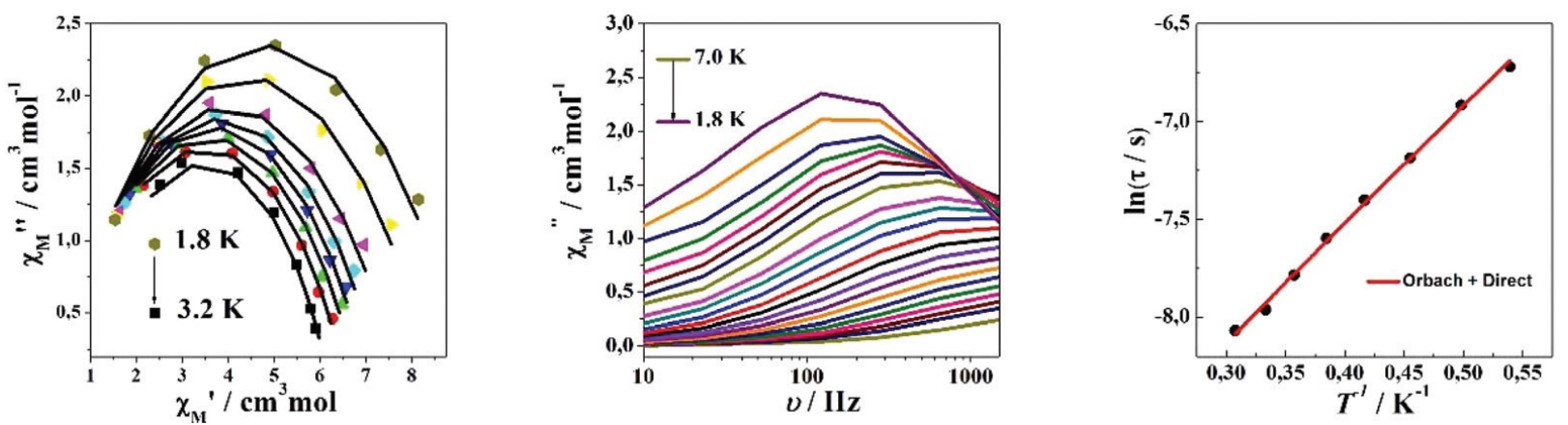

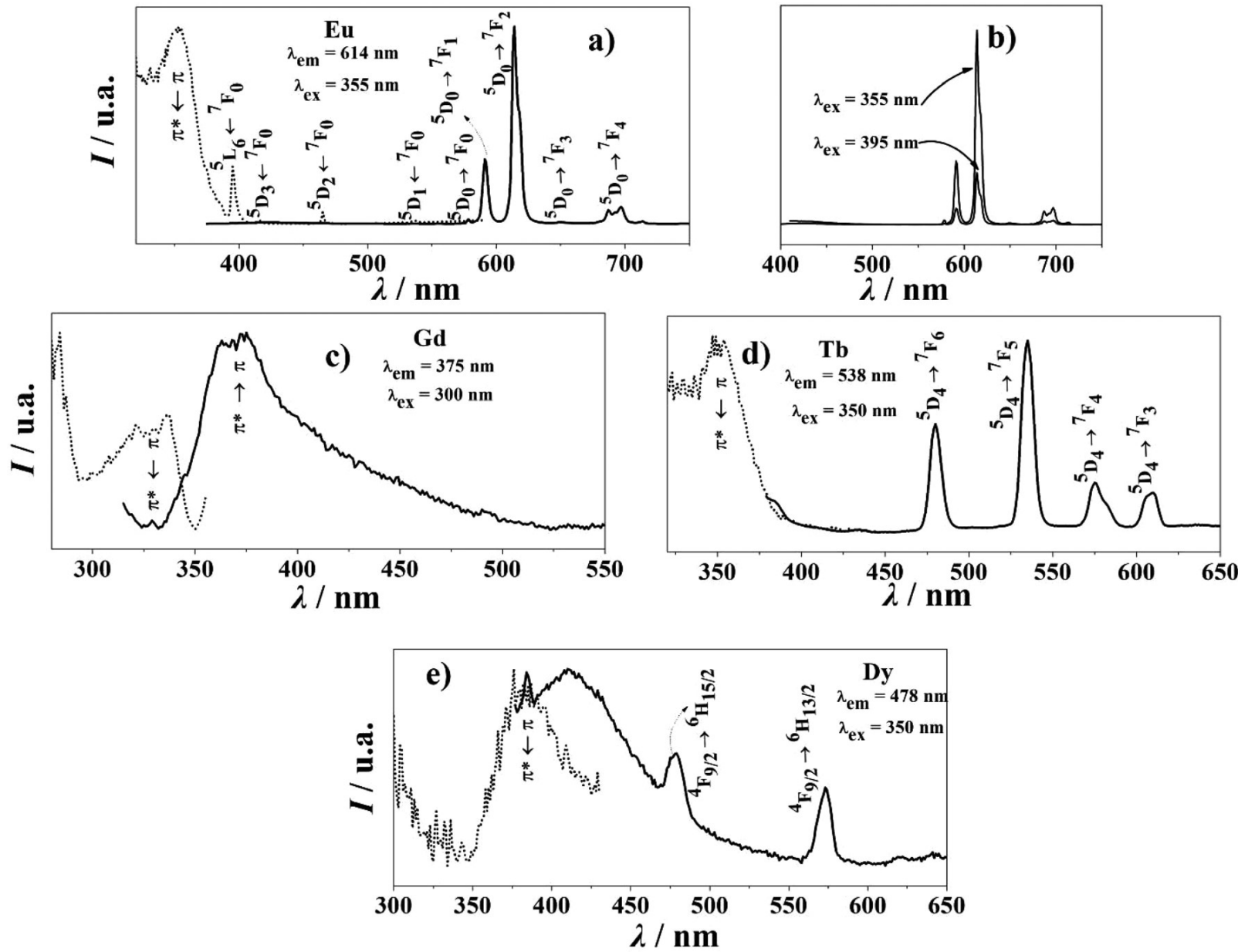

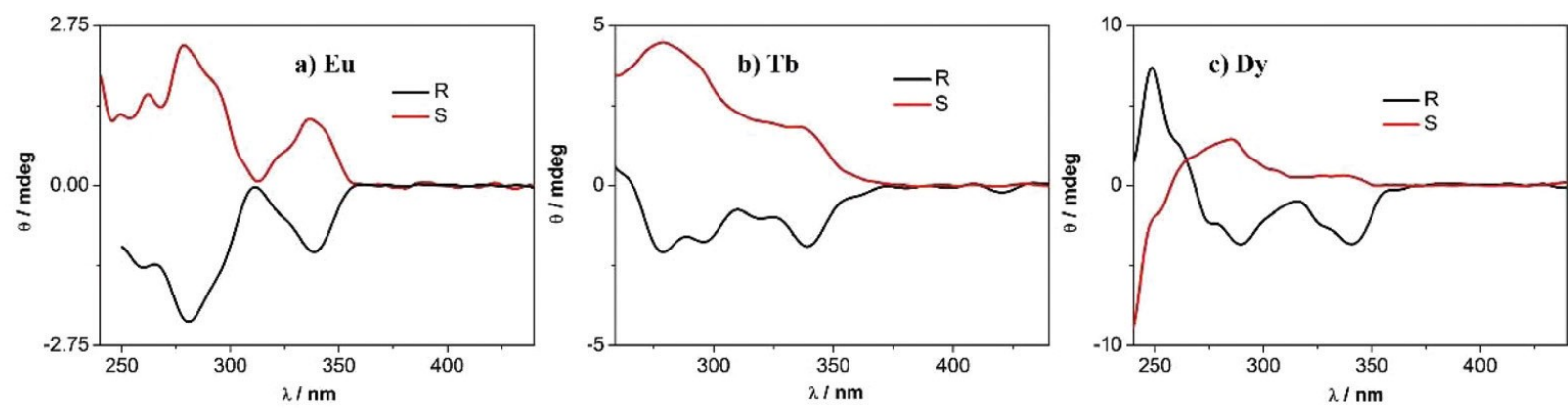

545 
549

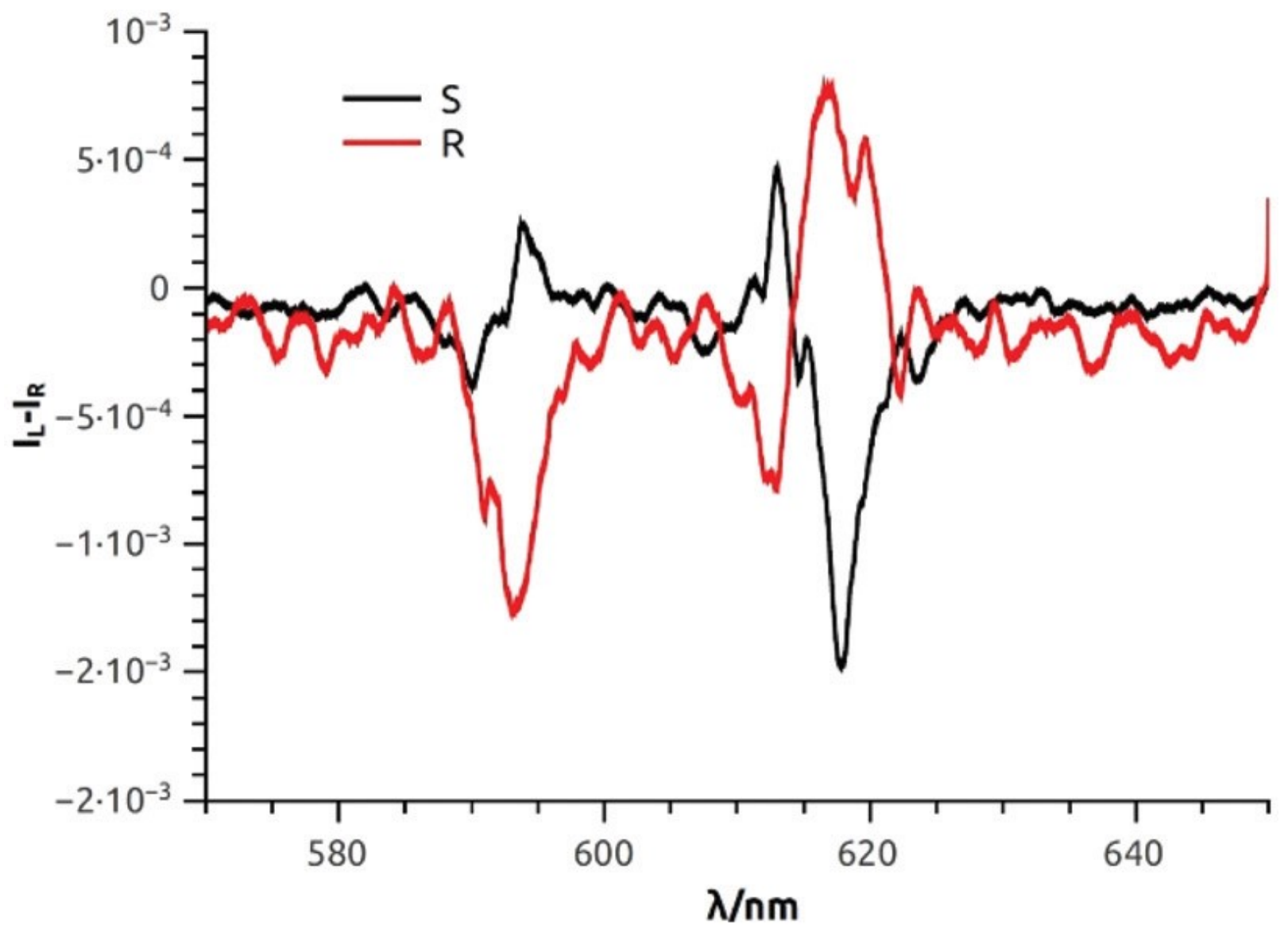

550

551

552 
553 Table 1 Selected bond distances $(\AA)$ and angles $\left(^{\circ}\right)$ for R/S-1, 3 and 4

\begin{tabular}{|c|c|c|c|c|c|c|}
\hline Bond/angle & $S-1$ & $R-1$ & $5-3$ & $R-3$ & $5-4$ & $R-4$ \\
\hline Ln1-I.n2 & $3.919(6)$ & $3.946(4)$ & $3.9174)$ & $3.917(5)$ & $3.902(1)$ & $3.904(4)$ \\
\hline $\mathrm{Ln} 1-\mathrm{O} 1$ & $2.355(6)$ & $2386(6)$ & $23395)$ & $23706)$ & $2.337(7)$ & $2345(6)$ \\
\hline $\operatorname{Ln} 1-03$ & $2.518(6)$ & $2560(6)$ & $2.499(5)$ & $2.544(5)$ & $2.496(6)$ & $2.538(5)$ \\
\hline $\mathrm{Ln} 1-\mathrm{O} 4$ & $2.506(6)$ & $2486(6)$ & $2.478(5)$ & $2.459(5)$ & $2.455(7)$ & $2.451 / 6)$ \\
\hline $\mathrm{Ln1}-0 \mathrm{~s}$ & $2.398(7)$ & $2389(5)$ & $2.352(5)$ & $2.357(5)$ & $2336(9)$ & $2373(5)$ \\
\hline $\operatorname{Ln} 1-07$ & $2.37(7)$ & $2368(5)$ & $2.335(5)$ & $2.551(5)$ & $2.332(7)$ & $2335(5)$ \\
\hline L.n1-09 & $2.436(8)$ & $2509(7)$ & $2.410(5)$ & $2.482(6)$ & $2399(9)$ & $2.476(6)$ \\
\hline $\operatorname{Ln} 1-010$ & $2.513(6)$ & $2466(6)$ & $2.4965)$ & $2.443(6)$ & $2.494(8)$ & $2.422(6)$ \\
\hline $\mathrm{Ln} 1-\mathrm{N1}$ & $2.644(9)$ & $2615(7)$ & $2.618(6)$ & $2.5877)$ & $2.598(9)$ & $2.583(7)$ \\
\hline Ln1-N2 & $2.5 \mathrm{~A}(\mathrm{~A})$ & $2611 / 7)$ & $2.548(5)$ & $2.585(7)$ & $2.545(8)$ & $2.557(7)$ \\
\hline $\mathrm{Lin}-\mathrm{O} 2$ & $2.373(7)$ & $237 A(7)$ & $2.56(5)$ & $2353(5)$ & $2344(8)$ & $2.334(5)$ \\
\hline $\mathrm{L}+\mathrm{n}-\mathrm{O} 3$ & $2.365(7)$ & $2369(5)$ & $2348(5)$ & $2335(5)$ & $2.331 / 7)$ & $2.326(5)$ \\
\hline $\operatorname{Lin} 2-06$ & $2.405(7)$ & $2365(6)$ & $2367(5)$ & $23375)$ & $2336(9)$ & $2.334(5)$ \\
\hline $\operatorname{Lin} 2-07$ & $2.57(6)$ & $2522(6)$ & $2.552(5)$ & $2.504(5)$ & $2.538(6)$ & $2.495(6)$ \\
\hline $\mathrm{L} n-\mathrm{O} A$ & $2.482(6)$ & $2500(6)$ & $2.461(5)$ & $2.481(5)$ & $2.452(7)$ & $2.454(5)$ \\
\hline $\mathrm{Ln} 2-011$ & $2.512(7)$ & $2432(7)$ & $2.484(6)$ & $2.410(7)$ & $2.462(8)$ & $2.406 / 5)$ \\
\hline $\mathrm{L} n-012$ & $2.454(8)$ & $2506(6)$ & $2.443(6)$ & $2.497(5)$ & $2.424(9)$ & $2.501(6)$ \\
\hline $\mathrm{L} n 2-\mathrm{N3}$ & 2.609 $(8)$ & $2637(6)$ & $2.5827)$ & $2.6276)$ & $2.582(8)$ & $2.591(6)$ \\
\hline $\mathrm{Ln} 2-\mathrm{N}_{4}$ & $2.605(8)$ & $2570 / 7)$ & $2.581(7)$ & $2.552(5)$ & $2.556(9)$ & $2.536(7)$ \\
\hline Ln1-03-Ln2 & $107.9(2)$ & $106.1(2)$ & $100.8(2)$ & $106.7(2)$ & $107.8(2)$ & $106.7 / 2$ \\
\hline $\mathrm{Ln} 1-07-\mathrm{Ln} 2$ & $105.7(2)$ & $107.6(2)$ & $106.5(2)$ & $100.5(2)$ & $106.4(2)$ & $107.9(2)$ \\
\hline
\end{tabular}

